\title{
Das neue Pflichtpraktikum an Handelsakademien - Was berichten Schüler/innen über Umsetzung und Kompetenzerwerb?
}

\author{
Christiane Schopf (iD $\cdot$ Birgit Aflenzer $\cdot$ Manuela Glas
}

Angenommen: 3. September 2019 / Online publiziert: 13. September 2019

(C) Der/die Autor(en) 2019

Zusammenfassung Pflichtpraktika sind in den Curricula österreichischer berufsbildender Schulen bereits seit Langem verankert. Eine Ausnahme stellten bisher die kaufmännischen Schulen dar. Mit dem Lehrplan 2014 wurde nun aber auch in diesem Schultyp eine verpflichtende Praxisphase eingeführt, um die Employability der Absolvent/inn/en zu stärken. Mit dieser Innovation sind umfangreiche Erwartungen verbunden. Es stellt sich die Frage, inwieweit diese tatsächlich eingelöst werden können. Erstaunlicherweise sind die Lernprozesse, die sich im Rahmen derartiger Praxisphasen bzw. durch die Verbindung von schulischem und betrieblichem Lernen vollziehen, bisher nur wenig wissenschaftlich erforscht. Ziel dieses Beitrags ist es vor diesem Hintergrund, im Rahmen einer Interviewstudie zu untersuchen, wie Handelsakademie-Schüler/innen die derzeitige Umsetzung des Pflichtpraktikums in Bezug auf Vorbereitungs-, Durchführungs- und Nachbereitungsphase erleben und welche konkreten Beiträge zum eigenen Kompetenzerwerb sie den einzelnen Phasen zuschreiben. Auf Basis der Erkenntnisse werden Handlungsempfehlungen für die gezielte Weiterentwicklung der Konzeption und Implementation des Pflichtpraktikums abgeleitet.

Schlüsselwörter Pflichtpraktikum · Betriebspraktikum · Portfolio · Reflexion · Berufliche Handlungskompetenz · Handelsakademie

C. Schopf $(\bowtie) \cdot$ B. Aflenzer $\cdot$ M. Glas Institut für Wirtschaftspädagogik, Department für Management, Wirtschaftsuniversität Wien, Welthandelsplatz 1, 1020 Wien, Österreich

E-Mail: christiane.schopf@wu.ac.at 


\title{
The new compulsory internship at business colleges - What students say about implementation and competence development
}

\begin{abstract}
Compulsory internships have long been part of the curricula of most Austrian vocational schools. However, only with the 2014 curriculum revision, a compulsory internship to strengthen the graduates' employability has also been established at business colleges. Considerable expectations are associated with this innovation. Hence, the question arises in how far these expectations can be met. Even more so because there is still a lack of research concerning the learning processes associated with internships and with the combination of school and workplace learning, respectively. Thus, this paper presents the results of an interview study exploring how business college students experience the implementation of the compulsory internship. Research interests are preparation and realisation of as well as reflection on these internships and which concrete contributions to their competence development students attribute to each of these phases. Concluding, suggestions for the further development of the concept as well as the implementation of the compulsory internship at business colleges are provided.
\end{abstract}

Keywords Compulsory Internship · Business Internship · Portfolio · Reflection · Professional Competence $\cdot$ Business College

\section{Stand der Forschung und Zielsetzung des Beitrags}

Die Curricula österreichischer berufsbildender Schulen sehen neben der schulischen Ausbildung die Absolvierung eines Praktikums vor. Eine Ausnahme stellten bisher die kaufmännischen Schulen dar, in denen praktische Übungen lediglich in sogenannten Übungsfirmen stattfanden. Diese Lücke wurde mit der Novellierung des Lehrplans 2014 geschlossen. Ab dem Schuljahr 2014/15 sind nun auch alle Schüler/ innen an Handelsakademien (HAK), Handelsschulen (HAS) sowie Aufbaulehrgängen dazu verpflichtet, im Rahmen ihrer Ausbildung ein facheinschlägiges Praktikum in einem Unternehmen oder einer Organisation ihrer Wahl zu absolvieren.

An diese Innovation im kaufmännischen Schulwesen sind vielfältige Erwartungen geknüpft. Laut Lehrplan sollen die folgenden Ziele erreicht werden: Umsetzung der in der Schule erworbenen Kompetenzen in der Berufsrealität, Einblick in die Organisation von Unternehmen, Kenntnis der Rechte und Pflichten von Arbeitnehmer/ inne/n und Arbeitgeber/inne/n, korrektes und selbstsicheres Verhalten gegenüber Vorgesetzten und Kolleg/inn/en, positive Grundhaltung zu Arbeitsleben und Beruf sowie unternehmerisches Denken und Handeln. ,Neben fachlichen sollen auch soziale und personale Kompetenzen erworben werden“ (Lehrplan der Handelsakademie 2014, S. 153). Ergänzend dazu wird im Durchführungserlass zum Pflichtpraktikum (2015) einerseits angeführt, dass die Schüler/innen im Praktikum Erfahrungen sammeln sollen, die zu einem besseren Verständnis der Unterrichtsinhalte führen, andererseits, dass das Praktikum den späteren Berufseinstieg erleichtern soll.

Die vorliegenden Evaluationsergebnisse aus anderen berufsbildenden Schultypen sowie der freiwilligen Praktika an HAK und HAS geben allerdings Anlass 
zu Zweifeln, inwieweit diese Erwartungen tatsächlich eingelöst werden können. So zeigt eine breit angelegte Studie von Eichmann et al. (2011), in der sowohl Pflicht- und freiwillige Praktika von Schüler/inne/n und Studierenden als auch Graduiertenpraktika in den verschiedensten Bereichen untersucht wurden, dass sich im Bereich wirtschaftlicher Berufe die Praktikumssuche am schwierigsten gestaltet und gleichzeitig die Zufriedenheit der Praktikant/inn/en am geringsten ausfällt. Aus dem Projekt PEARL der Universität Innsbruck (Ostendorf 2016, 2017), in dem 59 Schüler/innen an HTL (Höhere Technische Lehranstalt) und HLT (Höhere Lehranstalt für Tourismus) ihr Lernen im Praktikum aus der Innensicht erforschten, wird deutlich, dass zwischen den Branchen große Unterschiede bestehen. In gastronomischen Praktika findet meist eine weitgehende Integration der Praktikant/inn/en in den Arbeitsprozess statt. Wie auch eine von Ammann und Thoma (2011) durchgeführte quantitative Befragung von 444 Schüler/inne/n aus drei humanberuflichen Schulen in Tirol, die ihr Praktikum zumeist im Tourismus absolvierten, belegt, empfindet die überwiegende Mehrheit dieser Schüler/innen die ihnen übertragenen Aufgaben als angemessen, was zu einer sehr hohen Zufriedenheit mit dem Praktikum führt. In technischen oder kaufmännischen Praktika ist eine unmittelbare Integration in den Arbeitsprozess hingegen häufig nicht möglich. Hier stehen daher eher das Zeigen, Anleiten und Begleiten von Arbeit im Vordergrund (Ostendorf 2016, 2017). So ergibt auch eine quantitative Befragung von $325 \mathrm{HAS}$ - und HAK-Schüler/inne/n im Burgenland (Schnedl 2011), die freiwillig ein Betriebspraktikum absolvierten, dass oft nur Hilfstätigkeiten ausgeführt werden durften. Aus den Ergebnissen dieser Studie ist abzuleiten, dass im Rahmen eines kaufmännischen Praktikums die sozialen Kompetenzen gestärkt und die eigene Persönlichkeit weiterentwickelt werden können und dass die Schüler/innen Einblicke in die berufliche Wirklichkeit erhalten, die eine Reflexion über die eigene berufliche Zukunft ermöglichen, dass aber eine Kompetenzsteigerung in fachlicher Hinsicht nur sehr eingeschränkt erfolgt.

Eine erste bundesweite Evaluierung des neuen Pflichtpraktikums an kaufmännischen Schulen wurde von Lachmayr und Mayerl (2017) durchgeführt. Dabei wurden 2951 HAK- und HAS-Schüler/innen befragt, wovon sich $73 \%$ zum Zeitpunkt der Befragung im Praktikum befanden oder dieses bereits abgeschlossen hatten. Die Studie belegt, dass die Intention des Pflichtpraktikums durchaus positive Resonanz durch die Schüler/innen erfährt. Eine Faktorenanalyse ergab drei Faktoren, die für die Bewertung des Pflichtpraktikums zentral sind: Die größte Bedeutung kommt den durchgeführten Tätigkeiten zu; daneben sind die Bezahlung und die Rahmenbedingungen wie Arbeitszeiten und Erreichbarkeit relevant. Von $41 \%$ der Befragten wurden alle drei Dimensionen sehr gut, von $13 \%$ hingegen schlecht bewertet. $14 \%$ schätzten Tätigkeiten und Rahmenbedingungen als gut, die Bezahlung aber als sehr schlecht ein; umgekehrt schätzten $32 \%$ zwar Bezahlung und Rahmenbedingungen als sehr gut, die Tätigkeit jedoch als weniger gut ein. Immerhin $65 \%$ der Schüler/ innen fühlten sich im Praktikum angemessen gefordert, der Rest eher unter- als überfordert. Ein Wissenstransfer von der Schule ins Praktikum scheint bei vielen Lernenden zumindest manchmal gelungen zu sein, umgekehrt war dies in etwas geringerem Ausmaß der Fall. Die Unterstützung seitens der Schule in der Vorbereitungsphase wurde von den Schüler/inne/n eher kritisch bewertet und lediglich 
etwa ein Drittel der Schüler/innen gab an, dass die Praxiserfahrungen gemeinsam im Unterricht reflektiert wurden.

Insgesamt deuten die bisherigen Befunde darauf hin, dass Praktika im kaufmännischen Schulwesen mit spezifischen Herausforderungen verbunden sind und ihre Bewertung durch die Schüler/innen sowie ihr Lernpotential je nach konkretem Tätigkeitsbereich stark variieren können. Anzunehmen ist, dass die Nutzung des vorhandenen Lernpotentials durch die Art der Einbettung des Praktikums ins Gesamtcurriculum, d.h. durch die Gestaltung der Vorbereitung, Begleitung und Nachbereitung, beeinflusst wird.

Die Studie von Lachmayr und Mayerl (2017) bietet einen sehr guten Überblick über die Rahmenbedingungen des Pflichtpraktikums an kaufmännischen Schulen sowie dessen aktuelle Umsetzung und Bewertung durch die Schüler/innen. Der Fokus liegt hier allerdings auf einer statistisch-deskriptiven Darstellung inkl. Vergleich zwischen HAK und HAS sowie zwischen den Bundesländern. In Hinblick auf den Kompetenzerwerb wurde lediglich global erhoben, inwieweit es den Schüler/inne/n nach eigenen Angaben gelingt, einen Transfer zwischen den beiden Lernorten Schule und Betrieb herzustellen. In Bezug auf die von Schnedl (2011) berichteten - ebenfalls quantitativen - Befunde zum Kompetenzerwerb in freiwillig absolvierten Betriebspraktika ist zu hinterfragen, inwieweit diese unmittelbar auf das Pflichtpraktikum übertragbar sind, da die Motive der Praktikant/inn/en möglicherweise differieren und im Falle freiwilliger Praktika keinerlei Begleitung durch die Schule erfolgt. Die Relevanz einer schulischen Vor- und Nachbereitung für die Kompetenzentwicklung blieb in beiden Studien weitgehend unberücksichtigt.

Vor diesem Hintergrund verfolgt der vorliegende Beitrag das Ziel, genauer zu erfassen, wie HAK-Schüler/innen die derzeitige Umsetzung des Pflichtpraktikums in Bezug auf Vorbereitungs-, Durchführungs- und Nachbereitungsphase erleben und welche konkreten Beiträge zum eigenen Kompetenzerwerb sie den einzelnen Phasen zuschreiben. Um die Schüler/innen selbst zu Wort kommen zu lassen, wurde eine qualitative Interviewstudie durchgeführt. Als Hintergrund für die Darstellung und Interpretation der Ergebnisse wird im Folgenden zunächst die curriculare Ausgestaltung des Pflichtpraktikums an HAK näher erläutert. In Abschn. 3 wird in der gebotenen Kürze auf die zugrundeliegenden Annahmen über den Kompetenzerwerb durch Praktika eingegangen. Die Studie selbst wird in den Abschn. 4 und 5 präsentiert. Aus den Befunden werden schließlich in Abschn. 6 konkrete Handlungsempfehlungen für die Umsetzung des Pflichtpraktikums abgeleitet.

\section{Zur curricularen Ausgestaltung des Pflichtpraktikums an Handelsakademien}

\subsection{Praktikumsform}

Der Begriff des Praktikums ist gesetzlich nicht definiert und wird in Rechtsprechung, Literatur und Praxis uneinheitlich verwendet. Meist werden drei Erscheinungsformen unterschieden (Bundesministerium für Arbeit, Soziales und Konsumentenschutz 2017; Przeszlowska 2015): Bei einem Volontariat stehen die Erwei- 
terung und Anwendung von Kenntnissen in der Praxis im Vordergrund. Es handelt sich also um ein Ausbildungsverhältnis, das freiwillig eingegangen wird. Volontäre/ Volontärinnen unterliegen keiner persönlichen Abhängigkeit und keiner Arbeitsverpflichtung und haben dementsprechend auch keinen Entgeltanspruch. Ein Ferialarbeitsverhältnis hingegen ist ein Arbeitsverhältnis, das zum Zwecke des Geldverdienens und nicht primär zu Ausbildungszwecken aufgenommen wird. In Abgrenzung dazu sind Pflichtpraktika curricular verankert, stellen also einen integralen Bestandteil einer (hoch-)schulischen Ausbildung dar. Sie müssen hinsichtlich Inhalt und Dauer der jeweiligen Ausbildungsordnung entsprechen und sollen den theoretischen Unterricht durch praktische Erfahrungen ergänzen. Pflichtpraktika können sowohl als Ausbildungs- als auch als Arbeitsverhältnis ausgestaltet sein. Laut Lehrplan und Durchführungserlass ist das im Rahmen der HAK-Ausbildung vorgesehene Pflichtpraktikum in der Regel in Form eines Arbeitsverhältnisses zu absolvieren (Lehrplan der Handelsakademie 2014; Durchführungserlass zum Pflichtpraktikum 2015).

Aus didaktischer Perspektive können Praktika nach Ostendorf (2007) hinsichtlich der drei Dimensionen Grad der didaktischen Strukturierung, Fokus auf Lernen oder Arbeiten sowie zeitliche Dauer typologisiert werden. Berufsvorbereitende Praktika sind meist sehr kurze „Schnupperpraktika“, die hauptsächlich der Berufsfindung dienen. Demgegenüber stehen bei erfahrungsergänzenden Praktika die Verbindung von schulischen und betrieblichen Erfahrungen und der Erwerb praktischer Fähigkeiten im Zentrum. Diese sind daher umfangreicher, meist stärker didaktisch strukturiert und verknüpfen Lernen und Arbeiten. Während diese beiden Formen einem pädagogischen Kontext zuzuordnen sind, sind Berufseinstiegspraktika in einem ökonomischen Kontext zu sehen. Sie sollen es erleichtern auf dem Arbeitsmarkt Fuß zu fassen. Das Pflichtpraktikum an HAK ist primär als erfahrungsergänzendes Praktikum zu klassifizieren; ihm werden aber auch Funktionen der beiden anderen Praktikumsformen zugeschrieben. Ostendorf spricht in diesem Zusammenhang von „Betriebspraktikum“ und definiert dieses als ,zeitlich befristetes mehr oder weniger didaktisch strukturiertes Lern- und Arbeitsverhältnis in einem Unternehmen“ (Ostendorf 2007, S. 166).

\subsection{Vorgaben zu Umfang, Inhalt und Umsetzung}

Das Pflichtpraktikum an HAK umfasst laut Lehrplan 300 Arbeitsstunden und ist damit vom Umfang her im Vergleich zu anderen berufsbildenden höheren Schulen am unteren Ende des Spektrums angesiedelt. Empfohlen wird die Absolvierung in den Hauptferien zwischen II. und IV. Jahrgang, unter gewissen Voraussetzungen können auch Nebenbeschäftigungen angerechnet werden. Das Praktikum muss facheinschlägig sein, d.h. dem Bildungsziel der Schulform entsprechen (Lehrplan der Handelsakademie 2014; Durchführungserlass zum Pflichtpraktikum 2015; Bundesministerium für Bildung o.J.).

Die Organisation eines geeigneten Praktikumsplatzes liegt grundsätzlich in der Eigenverantwortung der Schüler/innen. Die Schule soll jedoch unterstützend tätig werden. Aufgabe der Schule ist es jedenfalls, die Schüler/innen frühzeitig über das Pflichtpraktikum zu informieren, sie vorab über ihre Rechte und Pflichten als Praktikant/inn/en aufzuklären und bei Schwierigkeiten Hilfestellung zu bieten. Das 
Praktikum ist in den betriebswirtschaftlichen Gegenständen, insbesondere im Gegenstand „Business Behaviour“ vor- und nachzubereiten. Der Lehrplan listet dazu folgende Inhalte auf: 3. Semester: „Angeleitete Vorbereitung und Organisation des Pflichtpraktikums (Auswahl eines geeigneten Unternehmens, Erstellen von Bewerbungsunterlagen, Vorstellungsgespräch, Zeitmanagement), Dokumentation durch ein Portfolio“; 8. Semester: „Nachbereitung der Erfahrungen aus dem Pflichtpraktikum unter den Gesichtspunkten von Arbeitsplatzbeschreibung, Tätigkeitsfelder, Rechtsform, Organisation, Produktpalette, rechtliche Rahmenbedingungen des Dienstverhältnisses“ (Lehrplan der Handelsakademie 2014, S. 13/15). Die Lehrkräfte dieses Gegenstands werden auch als zuständig benannt, mit den Praxisbetrieben Kontakt zu halten (Lehrplan der Handelsakademie 2014; Durchführungserlass zum Pflichtpraktikum 2015).

Sowohl die geleisteten Stunden als auch die Tätigkeiten sind in Form eines Praxisportfolios durch Firmenbestätigungen sowie eigene Dokumentation nachzuweisen. Laut Vorlage umfasst das Praxisportfolio darüber hinaus folgende Inhalte: Bewerbungsunterlagen, Unternehmensportrait, Beschreibung des Beschäftigungsverhältnisses und des Tätigkeitsprofils, Selbsteinschätzung hinsichtlich der erreichten Lehrziele sowie zusammenfassende verbale Darstellung der gewonnenen Erkenntnisse (Bundesministerium für Bildung 2014).

\section{Zugrundeliegende Annahmen über den Kompetenzerwerb durch Praktika}

Die Integration eines Pflichtpraktikums ins Curriculum verfolgt insgesamt das Ziel, die berufliche Handlungskompetenz der Absolvent/inn/en zu fördern. Im Folgenden soll geklärt werden, von welchem Verständnis beruflicher Handlungskompetenz und von welchen theoretischen Annahmen zum Kompetenzerwerb durch das Absolvieren von Praktika hier ausgegangen wird.

Nach Weinert (2001, S. 27f) können Kompetenzen verstanden werden als ,die bei Individuen verfügbaren oder durch sie erlernbaren kognitiven Fähigkeiten und Fertigkeiten, um bestimmte Probleme zu lösen, sowie die damit verbundenen motivationalen, volitionalen und sozialen Bereitschaften und Fähigkeiten um die Problemlösungen in variablen Situationen erfolgreich und verantwortungsvoll nutzen zu können“. Berufliche Handlungskompetenz wird Bader (1989) folgend als komplexes Konstrukt bestehend aus Fach-, Sozial- und Humankompetenz betrachtet, wobei Methoden-, Sprach- und Lernkompetenz als strukturelle Bestandteile dieser drei Komponenten gesehen werden. Der Erwerb beruflicher Handlungskompetenz setzt also die Verknüpfung von Kenntnissen, Fähigkeiten und Fertigkeiten mit Erfahrungen voraus.

Wirth (2013) konzeptionalisiert schulisches und betriebliches Lernen auf der Grundlage kognitionspsychologischer Schematheorien sowie Theorien mentaler Modelle - in Erweiterung des Ansatzes des Lernhandelns, den Tramm $(1992,1996)$ für die Übungsfirmenarbeit entwickelt hat - wie folgt: Es wird davon ausgegangen, dass kognitive Strukturen auf Basis subjektiver Erfahrungen in zwei Dimensionen verändert werden können. (1) Durch Abstraktion können Elemente zu Klassen zusam- 
mengefasst, im umgekehrten Fall können Klassen in Unterklassen zerlegt werden. (2) Durch Komplexion können einzelne Elemente in Relation zueinander gesetzt und zu einem Ganzen zusammengefügt werden, im umgekehrten Fall kann ein Ganzes in seine einzelnen Elemente zerlegt werden. Darüber hinaus ist die Flexibilität des Umgangs mit den gebildeten Schemata relevant, d.h. die Frage, in welchen Kontexten diese aktiviert und eingesetzt werden können. Während es beim schulischen Lernen primär um Abstraktion (exemplarischer Fälle zu Begriffen) und in weiterer Folge um Komplexion (einzelner Begriffe zu Theorien und Modellen) geht, stehen beim betrieblichen Lernen die Reduktion von Komplexität (betrieblicher Strukturen und Prozesse) sowie das Herstellen von Bezügen zu kognitiven Schemata im Vordergrund. Am Lernort Betrieb kann einerseits in der Schule begrifflich Abstrahiertes praktisch angewendet werden, andererseits können Praxiserfahrungen Ausgangspunkt für weitere schulische Lernprozesse sein. Für den Erwerb flexibler Schemata spielt Reflexion eine zentrale Rolle, denn ,,ein eigenständiges Bewegen in Abstraktions- und Komplexionshierarchien wird dadurch gefördert, dass die Lernenden unter Rückgriff auf ihre subjektiven Erfahrungen episodische Fälle (aus Schule und Betrieb) zu ihrem begrifflich abstrahierten Wissen durch Reflexion in Beziehung setzen“ (Wirth 2013, S. 12).

Für den Erwerb beruflicher Handlungskompetenz erscheint dementsprechend eine Kombination aus drei Lernakten notwendig (Ostendorf 2007, 2008): (1) Schulisches Lernen im Sinne eines theoretisch-systematischen Wissenserwerbs stellt eine notwendige Voraussetzung für die Mitarbeit in einem Betrieb dar. (2) Situatives Praxiswissen kann nur durch die Teilhabe an einer betrieblichen Praxisgemeinschaft (Community of Practice), also durch erfahrungsbasiertes, experimentelles Lernen im Betrieb, erworben werden. Dabei ist nach Lave und Wenger die unmittelbare Teilhabe zentral, um ,both absorbing and being absorbed in the ,culture of practice““ (Lave und Wenger 1991, S. 95). Die Lernenden müssen dazu von der Schüler/innenin die Mitarbeiter/innenrolle wechseln und sich als Noviz/inn/en in ein Expertenumfeld integrieren. (3) Um später auch in anderen (beruflichen) Kontexten verwertbares Wissen aufzubauen, müssen die konkreten Praxiserfahrungen anschließend reflektiert und in einen breiteren Kontext gestellt werden.

Um die Lernpotentiale des Praktikums optimal auszuschöpfen, erscheint es wichtig, Vorbereitung, Durchführung und Auswertung des Praktikums als didaktische Einheit zu konzipieren und zu realisieren (vgl. auch Loerwald 2011). Eine zentrale Rolle kann in diesem Zusammenhang dem Praxisportfolio zugeschrieben werden. Die Portfolioarbeit soll die Schüler/innen dazu motivieren und anleiten, sowohl ihren Arbeits- und Lernprozess als auch ihre Lernergebnisse zu dokumentieren und zu reflektieren und selbst Verantwortung dafür zu übernehmen (vgl. dazu Häcker 2005; Pfeifer und Kriebel 2007).

\section{Interviewstudie}

Vor diesem Hintergrund wurde im Rahmen der hier präsentierten qualitativen Studie folgenden Fragen nachgegangen: 
1. Wie erleben HAK-Schüler/innen die derzeitige Umsetzung des Pflichtpraktikums in Bezug auf Vorbereitungs-, Durchführungs- und Nachbereitungsphase?

2. Welche konkreten Beiträge zum eigenen Kompetenzerwerb schreiben sie den einzelnen Phasen zu?

Zur Erhebung der individuellen Sichtweisen der Schüler/innen wurden Einzelinterviews in Form von Leitfadeninterviews (Flick 2014) geführt. Im Leitfaden wurden in Anlehnung an das Verständnis von Helfferich (2014) unterschiedliche Frageformen kombiniert: Zum Einstieg wurden die Schüler/innen gebeten, ihren Lernerfolg auf einer fünfstufigen Skala einzuschätzen, ihre Angabe zu begründen und auszuführen, worin dieser Lernerfolg konkret bestand. Im Zentrum stand dabei nicht die Quantifizierung, sondern die Möglichkeit für die Befragten, zunächst frei über ihre Lernerfahrungen im Praktikum zu berichten und damit ihre eigenen Relevanzstrukturen zum Ausdruck zu bringen. Erst im Anschluss daran wurden anhand einer Reihe von Fragen mit engerem Antwortspielraum die drei Phasen Vorbereitung, Durchführung und Nachbereitung besprochen, um die Umsetzung des Praktikums und die sich daraus ergebenden Lerngelegenheiten aus Schülersicht möglichst detailliert zu erfassen. Abschließend wurden die Schüler/innen gebeten, die Einführung des Pflichtpraktikums insgesamt zu bewerten.

Die Befragung wurde in sieben Klassen an fünf verschiedenen niederösterreichischen HAK durchgeführt. Es wurde der III. Jahrgang ausgewählt, da die Absolvierung des Pflichtpraktikums ab dem II. Jahrgang empfohlen wird und für die höheren Jahrgänge zum Zeitpunkt der Erhebung (November/Dezember 2017) der neue Lehrplan noch nicht in Kraft war. Die Teilnahme der Schüler/innen war selbstverständlich freiwillig. Insgesamt konnten 20 Personen, 17 Schülerinnen und drei Schüler, die das Praktikum bereits absolviert hatten, für ein Gespräch gewonnen werden. Die Dauer der Interviews variierte je nach Auskunftsbereitschaft der Schüler/innen beträchtlich und lag zwischen 18 und $46 \mathrm{~min}$. Die Gespräche wurden mit Einverständnis der Befragten auf Tonband aufgezeichnet und anschließend vollständig, in Form der literarischen Umschrift (Höld 2009), transkribiert.

Die Auswertung des Datenmaterials erfolgte in Anlehnung an die inhaltlich strukturierende Inhaltsanalyse nach Mayring (2015). Die folgenden acht Hauptkategorien wurden deduktiv aus dem Forschungsinteresse abgeleitet:

- Vorbereitung (Aussagen, die sich auf Aktivitäten in der Schule zur Vorbereitung auf das Praktikum beziehen, z.B. Informationsveranstaltungen, Erstellung von Bewerbungsunterlagen, Simulation von Bewerbungsgesprächen, Besprechung des Portfolios)

- Stellensuche (Aussagen, die sich direkt auf die Suche nach einer Praktikumsstelle beziehen, d.h. auf das Herstellen von Kontakten und den Bewerbungsprozess)

- Lernerfolg im Rahmen der Vorbereitungsphase (Aussagen, aus denen explizit oder implizit erkennbar wird, welcher Lernerfolg bei der Vorbereitung auf das Praktikum in der Schule sowie bei der Stellensuche wahrgenommen wurde)

- Praktikum (Aussagen, die sich auf die Praktikumszeit im Unternehmen beziehen, z.B. auf Tätigkeiten, Erfahrungen mit Vorgesetzten, Kolleg/inn/en und Kund/inn/en, Schwierigkeiten) 
- Lernerfolg im Rahmen des Praktikums (Aussagen, aus denen explizit oder implizit erkennbar wird, welcher Lernerfolg während des Praktikums im Unternehmen wahrgenommen wurde)

- Nachbereitung (Aussagen, die sich auf Aktivitäten in der Schule zur Nachbereitung des Praktikums beziehen, inkl. Portfolioarbeit)

- Lernerfolg im Rahmen der Nachbereitungsphase (Aussagen, aus denen explizit oder implizit erkennbar wird, welcher Lernerfolg bei der Nachbereitung des Praktikums in der Schule wahrgenommen wurde)

- Gesamtbewertung des Pflichtpraktikums (Aussagen, die erkennen lassen, wie die Einführung des Pflichtpraktikums insgesamt bewertet wird)

Im ersten Schritt wurden die inhaltstragenden Textpassagen diesen Hauptkategorien zugeordnet. Im zweiten Schritt erfolgte eine Paraphrasierung, Generalisierung und Reduktion der Aussagen pro Hauptkategorie. Im letzten Schritt wurden aus dem reduzierten Material induktiv Unterkategorien gebildet. Diese stellen die Basis für die Beschreibung und Interpretation der Ergebnisse dar.

Im Rahmen der Interviews wurden die Schüler/innen auch gebeten, ihre Praxisportfolios für die Studie zur Verfügung zu stellen. Da nicht alle Befragten dazu bereit waren und einige das Portfolio noch gar nicht erstellt hatten, lagen letztlich nur neun Dokumente vor. Aus diesem Grund wurden die Praxisportfolios lediglich als Kontextmaterial verwendet, auf eine systematische Analyse wurde verzichtet.

\section{Ergebnisse}

Die Ergebnisse der Interviewstudie werden im Folgenden entlang der oben definierten Hauptkategorien dargestellt, anhand von prägnanten Schülerzitaten illustriert ${ }^{1}$ und, wo sinnvoll, auch quantifiziert.

In Hinblick auf die Vorbereitung des Pflichtpraktikums an den Schulen zeigte sich, dass die Lehrplanvorgaben in unterschiedlichem Ausmaß erfüllt werden. In sechs von sieben Klassen wurden im 3. Semester (Wintersemester des II. Jahrgangs) im Gegenstand Business Behaviour Bewerbungsunterlagen erstellt und Bewerbungstrainings durchgeführt. In allen Klassen wurde das Thema Bewerbungsmappe (erneut) im III. Jahrgang in Office Management und angewandter Informatik aufgegriffen, wobei hier der Fokus auf den Formerfordernissen lag. Dies entspricht zwar dem Lehrplan und wird von den meisten Schüler/inne/n auch als sehr hilfreich erachtet, viele bemängelten aber, dass die Erstellung der Bewerbungsunterlagen zu spät erfolgte, da diese bereits zu einem früheren Zeitpunkt für die rechtzeitige Bewerbung um einen Praktikumsplatz zur Verfügung hätten stehen müssen. Das Praxisportfolio wurde nur in zwei Schulen vorab besprochen. Für die Schüler/innen, die lediglich einen Hinweis auf das Erfordernis eines Portfolios erhielten, entstanden dadurch viele Unklarheiten. Auch die Aufklärung über Rechte und Pflichten von Praktikant/inn/en scheint an den Schulen in recht unterschiedlichem Umfang und Kontext

\footnotetext{
${ }^{1}$ Auf die Schülerzitate wird jeweils durch Angabe der aus Anonymitätsgründen chronologisch vergebenen Schülernummer hingewiesen, z. B. S5=Schüler/in Nr. 5.
} 
(in Business Behaviour, in Betriebswirtschaft oder auch im Rahmen externer Vorträge) zu erfolgen, was wohl auch damit zusammenhängt, dass diese Aufgabe im Lehrplan lediglich in den didaktischen Grundsätzen festgehalten ist. So fühlten sich einige Schüler/innen gut, andere nicht ausreichend informiert. Insgesamt lobten fünf Schüler/innen die gute Vorbereitung durch die Schule, während sich vier Schüler/ innen explizit beklagten, dass sie sich mehr Unterstützung erwartet hätten. Den im Zuge der Vorbereitung auf das Praktikum in der Schule erzielten Lernerfolg schätzten die Jugendlichen global als eher gering ein. Eine Weiterentwicklung von Methoden-, Sozial- und Humankompetenzen in Hinblick auf die Erstellung von Bewerbungsunterlagen sowie das Auftreten und Verhalten bei Bewerbungsgesprächen und am Arbeitsplatz kann dennoch aus vielen Aussagen abgeleitet werden: „Naja doch schon, wegen der Bewerbungsmappe vor allem, dass die perfekt ist und was da alles reingehört und wie die ausschauen soll, weil selber hätte ich das alles nicht gewusst so genau“ (S5); „Das mit dem Telefonieren hat mir eigentlich schon sehr viel gebracht, weil das ist halt wirklich gegliedert gewesen, wen rufe ich an, wann rufe ich an und was will ich.“(S16); ,Welchen Kleidungsstil man haben sollte und dass man nicht in zerrissenen Jeans und sowas zur Arbeit gehen sollte, die Umgangssprache, dass man höflich sein sollte sowieso, dass man aufmerksam sein soll, wenn irgendwer Hilfe braucht, dass man den unterstützt, und Ordnung hält"“ (S7).

Die Suche nach einem geeigneten Praktikumsplatz gestaltete sich nach Auskunft der Schüler/innen unterschiedlich schwierig. 16 der 20 Jugendlichen erhielten ihre Stelle letztlich über eine verwandte oder bekannte Kontaktperson im Unternehmen, also über persönliche Beziehungen. Dennoch verschickten viele Jugendliche mehrere Bewerbungen, fünf erhielten sogar mehrere Zusagen. Die Schule spielte im Suchprozess lehrplankonform nur eine untergeordnete Rolle. Die Lehrkräfte motivierten die Schüler/innen lediglich sich um eine Stelle zu bemühen. Fallweise wurden Hinweise auf Links, Inserate oder verfügbare Praktikumsplätze gegeben. Jedenfalls wurden aber Hilfestellungen angeboten, wenn es zu Problemen kam bzw. Schüler/ innen aktiv danach fragten. Von Seiten der Unternehmen wurden nur acht Praktikant/inn/en vorab zu einem Gespräch eingeladen; viele hatten daher vor Beginn des Praktikums keinen Kontakt zum Betrieb. Durch die mehr oder weniger selbständige Suche nach einem Praktikumsplatz konnten die Schüler/innen Erfahrungen für das spätere Berufsleben sammeln. Viele erkannten, wie schwierig und zeitintensiv es sein kann, einen geeigneten Job zu finden, besonders wenn keine persönlichen Kontakte zum Unternehmen vorhanden sind. Ein/e Befragte/r brachte diese Problematik wie folgt auf den Punkt: „Also ich habe das Gefühl gehabt, wenn man quasi keinen gekannt hat in einer Firma, ist es sehr schwer, dass du einen Platz kriegst“" (S9). Die Jugendlichen berichteten, dass sie Engagement, Ausdauer und Geduld beweisen mussten. So etwa Schüler/in 15: „Dass man halt wirklich dahinter sein muss, weil sonst kriegt man keinen Platz“ (S15). Als besondere Herausforderung erlebten viele den Umgang mit Absagen, die teilweise als verletzend empfunden wurden. Insgesamt konnten die Schüler/innen dadurch ihre Humankompetenzen ausbauen. Jene Praktikant/inn/en, die zu einem Bewerbungsgespräch eingeladen wurden, erhielten dadurch eine zusätzliche Gelegenheit ihre Sozialkompetenzen zu stärken.

Die befragten Schüler/innen absolvierten ihre Praktika in ganz unterschiedlichen Unternehmen. Das Spektrum der Tätigkeiten erstreckte sich von allgemeinen Bü- 
rotätigkeiten wie Kopieren, Sortieren, Postverteilung und Ablage über Telefondienst und Kundenbetreuung bis hin zu Arbeiten im Rahmen der Inventur, Lagerverwaltung oder Buchhaltung. Die ihnen übertragenen Aufgaben empfanden acht Schüler/ innen als vollständig, neun Schüler/innen als teilweise ihren fachlichen Qualifikationen entsprechend; der Rest fühlte sich unterfordert. Keiner der Befragten berichtete über Schwierigkeiten bei der Erledigung der Aufgaben. Die Praktikant/inn/en wurden ausreichend eingeschult und konnten bei Unklarheiten jederzeit nachfragen. Die Kolleg/inn/en wurden durchwegs als freundlich und hilfsbereit beschrieben. Alle Jugendlichen fühlten sich im Betrieb wohl und gut betreut. Dazu trug wesentlich bei, dass es in den meisten Fällen eine Ansprechperson gab und sie ins Team gut aufgenommen, wenn auch oft nicht vollständig integriert wurden. Kontakt zur Schule bestand während der Praktikumszeit nicht, war aus Sicht der Jugendlichen aber auch nicht nötig. Der von den Schüler/inne/n empfundene fachliche Lernerfolg hängt erwartungsgemäß mit der Art der ausgeübten Tätigkeit, weniger mit dem Unternehmen(sbereich), in dem das Praktikum absolviert wurde, zusammen. Praktikant/inn/en, die anspruchsvollere Tätigkeiten ausüben durften, konnten ihre Fachkompetenzen vertiefen. Dies war hingegen bei Praktikant/inn/en, die lediglich einfache, vorhersehbare, sich wiederholende Tätigkeiten ausübten, kaum oder gar nicht der Fall. Dementsprechend berichteten die Schüler/innen auch in sehr unterschiedlichem Ausmaß davon, dass sie Bezüge zum Unterrichtsstoff herstellen bzw. das in der Schule - insbesondere in den Gegenständen Betriebswirtschaft, Unternehmensrechnung, Business Behaviour sowie Office Management und angewandte Informatik - erworbene Wissen und Können im Betrieb nutzen konnten. Wo dies möglich war, zeigt sich deutlich das damit verbundene Kompetenzerleben. So beispielsweise Schüler/in 10: „Ich musste sogar jemandem helfen beim Serienbrief machen, weil er nicht wusste wie das geht, das fand ich cool“ (S10). Insgesamt ist jedoch festzuhalten, dass die Schüler/innen weniger im fachlichen, sondern vielmehr im sozialen und persönlichen Bereich eine Kompetenzsteigerung wahrnahmen. Durch die Arbeit im Team (mit Erwachsenen) sowie den Kontakt mit Kund/inn/en erwarben sie entsprechende Umgangsformen und Verhaltensweisen: ,wie das Zusammenarbeiten in Gruppen ist“ (S10), ,wie man mit den Kunden umgeht und spricht und auch, dass man ruhig bleibt, wenn der Kunde auszuckt“" (S5). In diesem Zusammenhang wurde auch ein Bewusstsein für die Konsequenzen von Fehlern entwickelt: „,dass man wirklich vieles kontrollieren muss und genau sein muss“ (S5); „In der Praxis, wenn man das dann wirklich macht mit dem Programm, wenn du es falsch eingibst, ist es blöd, weil der danach damit arbeitet, der hat dann ein Problem“ (S10). Viele Jugendliche sprachen zudem davon, disziplinierter, selbständiger und selbstbewusster geworden zu sein: ,Pünktlichkeit, also das war ich vorher nicht so gewohnt“ (S12); ,Wie ich mich organisieren soll, das habe ich gelernt“ (S13); ,Ich habe das Gefühl, ich bin sehr viel selbstbewusster geworden“" (S9).

Eine gezielte Nachbereitung des Praktikums in der Schule fand nach Angaben der Schüler/innen - zumindest bis zum Zeitpunkt der Erhebung - nicht statt. Nur einzelne Jugendliche berichteten von einem allgemeinen Erfahrungsaustausch. Dennoch sprachen 13 Schüler/innen davon, dass sie in der Schule von den Erfahrungen aus dem Praktikum profitierten, sei es in fachlicher Hinsicht, weil es ihnen danach leichter fiel im Unterricht einen Praxisbezug herzustellen - ,dass man sich beim Lernen 
von gewissen Themen leichter tut, da man einen wirklichen praktischen Bezug hat" (S17) - oder auf sozialer und persönlicher Ebene, z. B. durch eine Verbesserung der Selbstdisziplin sowie des Umgangs miteinander - „Nach dem Praktikum, da bin ich wirklich selbständiger geworden, auch in der Schule“ (S16). Die Notwendigkeit ein Praxisportfolio zu erstellen war zwar allen Schüler/inne/n bewusst, zum Zeitpunkt der Befragung hatten diese Aufgabe aber nur jene bereits erfüllt, denen ein frühzeitiger Abgabetermin seitens der Schule vorgegeben worden war. Lediglich eine Schülerin hatte freiwillig mit der Ausarbeitung begonnen. Die Durchsicht der zur Verfügung gestellten Portfolios ließ in Verbindung mit den Interviewaussagen der betreffenden Schüler/innen erkennen, dass die Qualität der Unterlagen einerseits von der Intensität der Begleitung durch die Lehrkraft beeinflusst wird und andererseits mit dem von den Schüler/inne/n wahrgenommenen Lernerfolg zusammenhängt. Die Relevanz und der Nutzen des Praxisportfolios erschlossen sich für die meisten Jugendlichen nicht, wie folgendes Zitat exemplarisch zeigt: „Das sind einfach nur Fragen, was soll man da großartig lernen“ (S9). Ein Lernerfolg wurde meist nur in Bezug auf die Recherche von Informationen zum Praxisbetrieb wahrgenommen. Eine einzige Schülerin schrieb dem Portfolio eine größere Bedeutung zu: Ihrer Ansicht nach leistete es einen wesentlichen Beitrag dazu, eine Verbindung zwischen Schule und Praxis herzustellen. Sie gab an, dass sie bei der Erstellung betriebswirtschaftliches Denken anwenden, Inhalte aus Office Management wiederholen und auch für den Deutschunterricht üben konnte. Dies kann zumindest als Indiz dafür betrachtet werden, dass das Instrument Portfolio grundsätzlich ein großes Lernpotential birgt.

Insgesamt befürworteten 17 der 20 Befragten die Existenz eines verpflichtenden Praktikums; 15 gaben an, dass sie auch auf freiwilliger Basis ein Praktikum absolviert hätten. Kritisiert wurde jedoch die hohe Zahl der nachzuweisenden Stunden. Alle Jugendlichen waren sich einig, dass sie durch das Praktikum einen guten, wenn auch begrenzten Einblick in die Arbeitswelt erhielten und es einen wichtigen Beitrag zu ihrer beruflichen Orientierung leistete. Einigen Schüler/inne/n gelang es auch sich ein erstes berufliches Netzwerk aufzubauen, das für weitere Praktika oder nach Abschluss der HAK nutzbar ist. Bei mehr als der Hälfte der Befragten hat sich die Einstellung zum Berufsleben, aber auch zur Schule verändert. Einige Aussagen weisen darauf hin, dass die Relevanz einer (höheren) wirtschaftlichen Ausbildung erkannt wurde, was zu einer größeren Wertschätzung des Schulbesuchs führte. So etwa Schüler/in 12: „Also vorher wollte ich arbeiten, also keine Schule mehr machen, arbeiten, damit ich mein Geld verdiene. Aber jetzt will ich Schule machen, auf jeden Fall“" (S12).

\section{Fazit und Handlungsempfehlungen}

Abschließend sollen die aus der Interviewstudie gewonnenen Erkenntnisse nochmals mit Bezug zu den Lehrplanvorgaben, den zugrundeliegenden Annahmen über den Kompetenzerwerb sowie den bisherigen empirischen Untersuchungen zusammengefasst werden, um daraus Handlungsempfehlungen für die Umsetzung der einzelnen Phasen des Pflichtpraktikums abzuleiten. 
Die Aussagen der Befragten belegen erwartungsgemäß, dass die Qualität der Praxiserfahrungen wesentlich von den tatsächlich ausgeübten Tätigkeiten sowie der Betreuung im Betrieb und die Qualität der Vor- und Nachbereitung vom Engagement der Lehrkräfte abhängen und folglich stark variieren. Wenngleich der Anteil der Praktikant/inn/en, die lediglich Hilfsarbeiten leisten durften, in den oben angeführten Studien schwankt, wird insgesamt die Problematik evident, dass es gerade im kaufmännischen Bereich an Praktikumsstellen mangelt, die dem Ausbildungsstand der Schüler/innen eines II. oder III. Jahrgangs HAK entsprechen. Die Lernenden erhalten nur bedingt die Möglichkeit, in der Schule begrifflich Abstrahiertes im Betrieb praktisch anzuwenden und entsprechende originäre Erfahrungen zu sammeln. Dies hat - wie die vorliegenden qualitativen Ergebnisse bestätigen - zur Folge, dass das Praktikum zwar einen wichtigen Beitrag zur Weiterentwicklung von Sozial- und Humankompetenzen zu leisten vermag, der Beitrag zur fachlichen Kompetenzentwicklung aber zumeist hinter den Erwartungen zurückbleibt.

Erfreulich ist, dass sich die Mehrheit der Befragten im Praktikumsbetrieb freundlich aufgenommen und gut betreut fühlt. Vollständig integriert in den Arbeitsprozess und in die Gemeinschaft werden die Jugendlichen Einzelberichten zufolge allerdings eher dann, wenn sie einem längerfristigen Nebenjob nachgehen. Dieser Befund gibt zu denken, da diese Praktikumsform in den Ordnungsmitteln derzeit nicht favorisiert und laut der Erhebung von Lachmayr und Mayerl (2017) auch nur von einem sehr geringen Anteil der Schüler/innen gewählt wird. Geht man davon aus, dass nur die vollständige Teilhabe an einer Community of Practice ein schrittweises Hineinwachsen in eine Praxiskultur und damit die Entwicklung vom Novizen zum Experten ermöglicht, stellt sich die Frage, ob diese Praktikumsform nicht stärker gefördert werden sollte. Das große Potential begleitender Praktika wird auch durch einen sehr erfolgreichen Hamburger Schulversuch belegt: „Das Zwei-Tage-Modell im Rahmen halbjährlicher Praktika erlaubt es den Betrieben, die Schülerinnen und Schüler über den sonst üblichen Gaststatus hinaus in die betrieblichen Abläufe zu integrieren. Diese Integration ist eine entscheidende Voraussetzung für die Erfahrung von Kompetenz, die ihrerseits das emotionale Fundament der entwickelten Lernmotivation darstellt.“ (Bastian et al. 2007, S. 256).

In Bezug auf die Vorbereitungsphase können folgende konkrete Verbesserungsvorschläge festgehalten werden: Die Gestaltung von Bewerbungsunterlagen sollte auf den Beginn des 3. Semesters vorverlegt werden, damit die Bewerbungen rechtzeitig verschickt werden können. Sinnvollerweise im 4. Semester sollten die Schüler/innen umfassend über ihre Rechte und Pflichten als Praktikant/inn/en aufgeklärt werden. Darüber hinaus wird empfohlen, die Lernenden im Vorfeld der Praktikumszeit zu einer strukturierten Beschäftigung mit dem Praktikumsbetrieb und dessen Umfeld sowie mit dem branchenspezifischen Fachwortschatz - etwa in Form von Recherchen, der Lektüre von Fachbeiträgen oder der Bearbeitung von Fallbeispielen - anzuleiten, um diesen einerseits die Entwicklung realistischer Erwartungen und Ziele zu ermöglichen, andererseits die spätere Einordnung der Praxiserfahrungen zu erleichtern. Hier sollten auch die Betriebe stärker in die Pflicht genommen werden, den Schüler/inne/n die Gelegenheit zu geben, sich bereits im Vorfeld, etwa im Rahmen eines Bewerbungsgesprächs und/oder einer Betriebsbesichtigung, ein Bild vom Unternehmen und dem zu absolvierenden Praktikum zu verschaffen. 
Da alle Studien unisono zeigen, dass die Schüler/innen bei der Suche nach einem geeigneten Praktikumsplatz sehr stark auf private Beziehungen angewiesen sind, womit unweigerlich die Benachteiligung von Jugendlichen, die über kein entsprechendes Netzwerk verfügen, einhergeht, sollten Unterstützungsangebote seitens der Schule ausgebaut werden. Die Vermittlung sollte jedoch nicht gänzlich durch die Schule übernommen werden, da den Interviewergebnissen zufolge bereits die selbständige Suche nach einem Praktikumsplatz einen Beitrag zur Kompetenzentwicklung leisten kann.

Erheblicher Verbesserungsbedarf ist in Hinblick auf die Nachbereitungsphase zu diagnostizieren, da die für den Kompetenzerwerb zentrale Reflexion der Praxiserfahrungen derzeit bedauerlicherweise viel zu kurz zu kommen scheint. Diese sollte so angelegt sein, dass sie den Lernenden vielfältige Möglichkeiten bietet, Bezüge zwischen ihren subjektiven Erfahrungen und ihren verfügbaren kognitiven Schemata herzustellen und die Praxiserfahrungen als Ausgangspunkt für weitere schulische Lernprozesse zu nutzen. Dies kann etwa durch gezielten Erfahrungsaustausch, die Ausarbeitung von Fallbeispielen oder die Gestaltung einer Praktikums-Messe gelingen. In diesem Zusammenhang sollten jedenfalls die Potenziale der Portfolioarbeit viel stärker ausgeschöpft werden. Wie bereits die Zahlen aus der Studie von Lachmayr und Mayerl (2017) vermuten ließen, machen auch die vorliegenden Ergebnisse deutlich, dass die Portfolioarbeit im Unterricht stärker angeleitet und eingefordert werden muss. Die Interviewaussagen zeigen eindrücklich, dass der Großteil der Schüler/innen Sinn und Zweck des Portfolios nicht erkennt. Zugleich legt jedoch die Sichtung der Portfolios den Schluss nahe, dass ein positiver Zusammenhang zwischen Qualität der Portfolioarbeit und insgesamt wahrgenommenem Lernerfolg besteht. Es wäre daher wichtig, den Schüler/inne/n frühzeitig die Bedeutung einer das Praktikum begleitenden Portfolioarbeit sowie auch den Nutzen des Portfolios als (Teil eines) Talent- oder Bewerbungsportfolio(s) (vgl. dazu Endres et al. 2008) $\mathrm{zu}$ vermitteln. Es wird empfohlen, bereits in der Vorbereitungsphase gemeinsam mit der Portfolioarbeit zu beginnen und die Schüler/innen anzuhalten, das Portfolio während der Praktikumszeit laufend fortzuführen und zeitnah abzuschließen. Um dies sicherzustellen, sollte zu Beginn des 5. Semesters ein Abgabetermin festgelegt und individuell Rückmeldung zum Produkt gegeben werden. Ein Rückgriff auf die Praxiserfahrungen erst im 8. Semester - wie derzeit im Lehrplan verankert - ist jedenfalls zu spät.

Insgesamt kann das Pflichtpraktikum als wichtige Innovation im Lehrplan der HAK bezeichnet werden, die von den Schüler/inne/n sehr positiv angenommen wird. Der besondere Wert liegt für die Jugendlichen in der Möglichkeit des zeitlich befristeten Wechsels von der Schüler/innen- in die Mitarbeiter/innenrolle, um einen ersten generellen Einblick in die Arbeitswelt zu erhalten (vgl. auch Beinke 2013). Um sein volles Potential zu entfalten, müsste das Praktikum allerdings von allen Beteiligten tatsächlich als Lernraum gedacht werden, der Vorbereitung, Begleitung und Nachbereitung umfasst. Diesbezüglich sei auf das erst kürzlich erschienene umfassende Werk von Ostendorf et al. (2018) verwiesen, das - im Sinne einer konnektivitätsorientierten Praktikumsdidaktik - zahlreiche Anregungen und Tools zur Unterstützung des Lernens sowohl von Seiten der Schule als auch der Betriebe beinhaltet. 
Wenngleich sich die Ergebnisse der vorliegenden Interviewstudie sowie die daraus abgeleiteten konkreten Handlungsempfehlungen spezifisch auf Konzeption und Implementation des Pflichtpraktikums an Handelsakademien beziehen, kann unseres Erachtens davon ausgegangen werden, dass diese auch für die Ausgestaltung anderer Formen schulischer Praxisphasen einige hilfreiche Hinweise liefern. Insbesondere sei nochmals festgehalten, dass alleine durch das Festschreiben eines verpflichtenden Praktikums im Curriculum noch wenig über die tatsächlich damit verbundenen Lerngelegenheiten ausgesagt ist, da diese ganz wesentlich von der Art der Eingliederung des Praktikanten/der Praktikantin im Betrieb sowie von Qualität und Intensität der vor- und nachbereitenden Maßnahmen abhängen. In diesem Zusammenhang sollte aufgrund der großen Bedeutung einer vollständigen Teilhabe an einer Community of Practice neben der typischen Form des „Ferialpraktikums“ auch die Option einer geringfügigen kontinuierlichen beruflichen Tätigkeit parallel zum Schulbesuch stärker berücksichtigt werden. Im Rahmen schulischer Praktika sollte der Anspruch an die fachliche Kompetenzentwicklung wohl generell nicht zu hoch gesetzt werden. Die Absolvierung eines Praktikums erscheint aber dennoch jedenfalls in Hinblick auf die Weiterentwicklung von Sozial- und Humankompetenzen lohnend.

Funding Open access funding provided by Vienna University of Economics and Business (WU).

Open Access Dieser Artikel wird unter der Creative Commons Namensnennung 4.0 International Lizenz (http://creativecommons.org/licenses/by/4.0/deed.de) veröffentlicht, welche die Nutzung, Vervielfältigung, Bearbeitung, Verbreitung und Wiedergabe in jeglichem Medium und Format erlaubt, sofern Sie den/die ursprünglichen Autor(en) und die Quelle ordnungsgemäß nennen, einen Link zur Creative Commons Lizenz beifügen und angeben, ob Änderungen vorgenommen wurden.

\section{Literatur}

Ammann, M., \& Thoma, M. (2011). Entwicklung eines Qualitätsmodells für Betriebspraktika: Erste empirische Einblicke. Wissenplus, 29, 61-66.

Bader, R. (1989). Berufliche Handlungskompetenz. Die berufsbildende Schule, 41, 73-77.

Bastian, J., Combe, A., Hellmer, J., \& Wazinski, E. (2007). Zwei Tage Betrieb - drei Tage Schule: Kompetenzentwickung in der Lernortkooperation an Allgemeinbildenden Schulen. Bad Heilbrunn: Julius Klinkhardt.

Beinke, L. (2013). Das Betriebspraktikum als Instrument der Berufsorientierung. In T. Brüggemann \& S. Rahn (Hrsg.), Berufsorientierung: Ein Lehr- und Arbeitsbuch (S. 262-269). Münster: Waxmann.

Bundesministerium für Arbeit, Soziales und Konsumentenschutz (2017). Rechtliche Situation von Praktikanten/Praktikantinnen in Österreich: Ein Leitfaden für die Absolvierung von Praktika in Betrieben in Österreich. Wien: Bundesministerium für Arbeit, Soziales und Konsumentenschutz.

Bundesministerium für Bildung (o.J.). Pflichtpraktikum an kaufmännischen Schulen: Informationsblatt für Schüler/innen. Wien.

Bundesministerium für Bildung (2014). Leitfaden Praxisportfolio HAK. Linz: Bundesministerium für Bildung.

Durchführungserlass zum Pflichtpraktikum (2015). Durchführung des Pflichtpraktikums an kaufmännischen Lehranstalten.

Eichmann, H., Saupe, B., Vogt, M., \& Scheiflinger, S. (2011). Praktika und Praktikanten/Praktikantinnen in Österreich: Empirische Analyse von Praktika sowie der Situation von Praktikanten/Praktikantinnen. FORBA-Forschungsbericht 4/2011. Wien: Forschungs- und Beratungsstelle Arbeitswelt.

Endres, W., Wiedenhorn, T., \& Engel, A. (2008). Das Portfolio in der Unterrichtspraxis: Präsentations-, Lernweg- und Bewerbungsportfolio. Weinheim: Beltz.

Flick, U. (2014). Qualitative Sozialforschung: Eine Einführung. Reinbek bei Hamburg: Rowohlt.

Häcker, T. (2005). Mit der Portfoliomethode den Unterricht verändern. Pädagogik, 57, 13-18. 
Helfferich, C. (2014). Leitfaden- und Experteninterviews. In N. Baur \& J. Blasius (Hrsg.), Handbuch Methoden der empirischen Sozialforschung (S. 559-574). Wiesbaden: Springer.

Höld, R. (2009). Zur Transkription von Audiodaten. In R. Buber \& H. H. Holzmüller (Hrsg.), Qualitative Marktforschung. Konzepte - Methoden - Analysen (S. 655-668). Wiesbaden: Gabler.

Lachmayr, N., \& Mayerl, M. (2017). Das Pflichtpraktikum an kaufmännischen Schulen. Erste bundesweite Evaluierung. Wien: Österreichisches Institut für Berufsbildungsforschung.

Lave, J., \& Wenger, E. (1991). Situated learning. Legitimate peripheral participation. Cambridge: Cambridge University Press.

Lehrplan der Handelsakademie (2014). BGBl. II Nr. 209/2014.

Loerwald, D. (2011). Das Schülerbetriebspraktikum - Betriebe als außerschulische Lernorte. In T. Retzmann (Hrsg.), Methodentraining für den Ökonomieunterricht II (S. 125-140). Schwalbach: Wochenschau.

Mayring, P. (2015). Qualitative Inhaltsanalyse: Grundlagen und Techniken. Weinheim: Beltz.

Ostendorf, A. (2007). Das Betriebspraktikum zwischen fruchtbarer Lernsituation und Disziplinierungstechnologie. In D. Münk (Hrsg.), Hundert Jahre kaufmännische Ausbildung in Berlin (S. 164-173). Opladen: Budrich.

Ostendorf, A. (2008). Betriebspraktika als Lernsituation gestalten. Didaktische Möglichkeiten und Grenzen. Wissenplus, 26, 18-22.

Ostendorf, A. (2016). Welche Lernräume nutzen BHS-Schüler/innen im Betriebspraktikum? - Erste Ergebnisse aus dem Sparkling Science Projekt PEARL. Wissenplus, 35, 12-16.

Ostendorf, A. (2017). Facetten und Bedeutung eines betrieblichen Praktikumsmentoring - Einsichten aus dem Projekt PEARL.bwp@, Profil 5, 1-11.

Ostendorf, A., Dimai, B., Ehrlich, C., \& Hautz, H. (2018). Den Lernraum Betriebspraktikum gemeinsam öffnen: Anspruch und Werkzeuge einer konnektivitätsorientierten Praktikumsdidaktik. Innsbruck: innsbruck university press.

Pfeifer, S., \& Kriebel, J. (2007). Lernen mit Portfolios. Neue Wege des selbstgesteuerten Arbeitens in der Schule. Göttingen: Vandenhoeck \& Ruprecht.

Przeszlowska, D. (2015). Praktikum: Ausbildungs- oder Arbeitsverhältnis? Zeitschrift für Arbeits- und Sozialrecht, 02/2015, 52-57.

Schnedl, K. (2011). Betriebspraktika an berufsbildenden Schulen - eine empirische Erhebung der Praktikumserfahrung von Schülerinnen und Schülern aus Handelsakademien und Handelsschulen. Masterarbeit. Wien: Wirtschaftsuniversität.

Tramm, T. (1992). Konzeption und theoretische Grundlagen einer evaluativ-konstruktiven Curriculumstrategie: Entwurf eines Forschungsprogramms unter der Perspektive des Lernhandelns. Dissertation. Göttingen: Georg-August-Universität Göttingen.

Tramm, T. (1996). Lernprozesse in der Übungsfirma. Rekonstruktion und Weiterentwicklung schulischer Übungsfirmenarbeit als Anwendungsfall einer evaluativ-konstruktiven und handlungsorientierten Curriculumstrategie. Habilitationsschrift. Göttingen: Georg-August-Universität Göttingen.

Weinert, F.E. (2001). Vergleichende Leistungsmessung in Schulen - eine umstrittene Selbstverständlichkeit. In I. F. E. Weinert (Hrsg.), Leistungsmessung in Schulen (S. 17-31). Weinheim: Beltz.

Wirth, K. (2013). Konnektive Didaktik zur Verknüpfung schulischen und betrieblichen Lernens - Kognitionspsychologische Grundlagen und didaktische Konsequenzen. bwp@, 24, 1-19. 